\title{
Susceptible-infected-susceptible epidemics on the complete graph and the star graph: Exact analysis
}

\author{
E. Cator* and P. Van Mieghem \\ Delft University of Technology, 2628 CN Delft, The Netherlands
}

(Received 2 August 2012; published 23 January 2013)

\begin{abstract}
Since mean-field approximations for susceptible-infected-susceptible (SIS) epidemics do not always predict the correct scaling of the epidemic threshold of the SIS metastable regime, we propose two novel approaches: (a) an $\varepsilon$-SIS generalized model and (b) a modified SIS model that prevents the epidemic from dying out (i.e., without the complicating absorbing SIS state). Both adaptations of the SIS model feature a precisely defined steady state (that corresponds to the SIS metastable state) and allow an exact analysis in the complete and star graph consisting of a central node and $N$ leaves. The $N$-intertwined mean-field approximation (NIMFA) is shown to be nearly exact for the complete graph but less accurate to predict the correct scaling of the epidemic threshold $\tau_{c}$ in the star graph, which is found as $\tau_{c}=\alpha \tau_{c}^{(1)}$, where $\alpha=\sqrt{\frac{1}{2} \log N+\frac{3}{2} \log \log N}$ and where $\tau_{c}^{(1)}=\frac{1}{\sqrt{N}}<\tau_{c}$ is the first-order epidemic threshold for the star in NIMFA and equal to the inverse of the spectral radius of the star's adjacency matrix.
\end{abstract}

DOI: 10.1103/PhysRevE.87.012811

PACS number(s): 89.75.Hc, 89.20.-a

\section{INTRODUCTION}

Epidemic processes on networks have gained considerable attention recently because they are regarded as a first-order description of how information in popular online social networks, such Hyves, Twitter, and Facebook, spreads. How many nodes (users) receive a particular message and which news item affects most users or with what intensity should news be spread to "infect" a considerable fraction of users in the network? These types of questions can be addressed (approximately) by a susceptible-infected-susceptible (SIS) epidemic process on an undirected graph $G(N, L)$, with $N$ nodes and $L$ links, characterized by a symmetric adjacency matrix $A$. In a SIS epidemic process, the viral state of a node $i$ at time $t$ is specified by a Bernoulli random variable $X_{i}(t) \in\{0,1\}: X_{i}(t)=0$ for a healthy node and $X_{i}(t)=1$ for an infected node. A node $i$ at time $t$ can be in one of the two states: infected, with probability $v_{i}(t)=\operatorname{Pr}\left[X_{i}(t)=1\right]$, or healthy, with probability $1-v_{i}(t)$ but susceptible to the infection. We assume that the curing process per node $i$ is a Poisson process with rate $\delta$ and that the infection rate per link is a Poisson process with rate $\beta$. Obviously, only when a node is infected, it can infect its direct neighbors, which are still healthy. The curing and infection Poisson processes are independent. The effective infection rate is defined by $\tau=\frac{\beta}{\delta}$. This is the general continuous-time description of the simplest type of a SIS epidemic process on a network.

Although a SIS epidemic process [1,2] is a simple abstraction of how a real virus spreads on a contact network, the SIS model is still too complex for general analytic treatment as shown in Ref. [3]. Only very few exact results exist, which are overviewed in Ref. [4]. Simon et al. [5] have employed graph-isomorphisms to reduce the $2^{N}$ states of the SIS Markov process and found that the complete graph, the star, and the household graph are exactly solvable with a reduced state space (without providing that exact solution).

*Faculty of Electrical Engineering, Mathematics and Computer Science, P.O. Box 5031, 2600 GA Delft, The Netherlands; E.A.Cator@tudelft.nl
Most papers investigating SIS epidemics on networks analyze the behavior approximately, often using a variant of a mean-field approach such as the Vespignani-Pastor-Satorras heterogeneous mean-field model [6] and the $N$-intertwined mean-field approximation (NIMFA) [3]. However, in spite of initial comparisons [7], the accuracy of mean-field SIS models has not been satisfactorily quantified yet.

Here, we assess the quality of NIMFA to the exact SIS process by considering two extreme graphs, the complete graph and the star graph. We start by relating the SIS model to a new model, called the modified SIS (MSIS). The MSIS model obeys the same evolution rules as the SIS model, except that when there is only one infected node in the system, this node is forbidden to heal. Hence, the infection will always survive, and there exists a nontrivial stationary measure for the MSIS. We can couple the SIS and MSIS models in the following way: the SIS process follows exactly the same evolution as the MSIS process, except when there is only one infected node. In that case, the SIS process has an extra exponential clock with rate $\delta$ running at this single infected node, independent of everything else. If this clock rings before the process in the MSIS model jumps to two infected nodes, the SIS process dies out, otherwise the SIS process follows the MSIS process. Thus, the epidemic process in the SIS model either equals that in the MSIS model or it has died out. The relevance of the stationary distribution of the MSIS model is now clear: If this distribution has a very small probability that only one node is infected, then the corresponding SIS process will be "metastable." If the probability that one node is infected is relatively large, then the SIS process will die out within a reasonable time. We will show in this paper that the MSIS model for the complete graph and for the star graph can be exactly determined, giving a detailed insight into the behavior of the SIS epidemic process for these two graphs. A surprising result is that if we choose our effective infection rate $\tau$ in the star graph to be equal to $C / \sqrt{N}$ (for some constant $C$ ), then we can show that, as $N \rightarrow \infty$, the SIS process will die out exponentially fast. In other words, the scaling of the epidemic threshold in the star is shown to be $\tau_{c}=\alpha \tau_{c}^{(1)}$, where $\alpha=\sqrt{\frac{1}{2} \log N+\frac{3}{2} \log \log N}$ and where $\tau_{c}^{(1)}=\frac{1}{\lambda_{1 \text {;star }}}=\frac{1}{\sqrt{N}}$ is 
the first-order epidemic threshold in NIMFA [8,9]. Since the correction factor $\alpha$ is small for realistic networks, simulations may fail to determine the correct scaling law for $\tau_{c}$.

In both the complete and star graph, the steady-state fraction $y_{\infty}$ of infected nodes, also called the prevalence, is determined and compared with the corresponding expression in NIMFA. In addition, we determine the precise scaling law for the number $M$ of infected nodes when $N$ grows large, which leads to an asymptotic scaling law for the epidemic threshold $\tau_{c}$ in the complete and star graph. These exact scaling laws allow us to verify the quality of the NIMFA epidemic threshold $\tau_{c}^{(1)}=\frac{1}{\lambda_{1}}$, where $\lambda_{1}$ denotes the largest eigenvalue of the adjacency matrix of the graph. While the mean-field type approximation as explained in Ref. [3] suggests very good results in the complete graph, as confirmed here, the mean-field method applied to the star graph seems less promising to determine the critical infection rate. However, for relatively large infection rates NIMFA predicts the steady-state fraction very well. When $\tau$ is large, the center of the star is (almost) always infected, which implies that the infection processes in the leaves of the star are almost independent. Just the independence assumption in infection probabilities, i.e., $\operatorname{Pr}\left[X_{i}=1, X_{j}=1\right]=\operatorname{Pr}\left[X_{i}=\right.$ $1] \operatorname{Pr}\left[X_{j}=1\right]$ is the only approximation made in NIMFA, which explains the excellent agreement of NIMFA with the exact results for large $\tau$.

While this paper concentrates on the MSIS model, earlier in Ref. [10], another approach to compute the metastable state was proposed. The SIS model was generalized to the $\varepsilon$-SIS model, where each node possesses an additional nodal selfinfection, modeled as an independent, third Poisson process with rate $\varepsilon$. Similarly to the MSIS model, the $\varepsilon$-SIS model has a precisely defined steady state. For small, but fixed $\varepsilon^{*}=$ $\frac{\varepsilon}{\delta}<\frac{1}{N}$, the corresponding steady state in the $\varepsilon$-SIS model was defined as the metastable state of the SIS process. The results derived in Sec. II for the complete graph in the MSIS model agree exceedingly well with the exact computation for the complete graph in the $\varepsilon$-SIS model [10].

After computing the MSIS process for the complete graph in Sec. II, we devote most of our efforts to the computation of the MSIS process on the star, which is considerably more complex than that of the complete graph. We deduce the exact Markov chain for the number of infected nodes in the star and present the exact MSIS steady state in the appendix. Unfortunately, these exact equations fail to provide physical insight, which has motivated to us to construct an asymptotic analysis for large $N$. The remarkable observation from our asymptotic analysis in Sec. III A is the discovery of two different, asymptotic scaling regimes with $N$ in the MSIS process in terms of the effective infection rate $\tau$. Finally, we compare the exact steady-state fraction of infected nodes in the star for various size $N$ with the exact, closed form Eq. (27) in NIMFA in Fig. 2. For finite $N$ and due to finite-size fluctuations, the precise onset of the infection is obscured most around the threshold $\tau_{c}$ and large variations in the order parameter (here, the steady-state fraction of infected nodes) occur (as illustrated in Fig. 2).

\section{SIS EPIDEMICS ON THE COMPLETE GRAPH}

We consider an SIS epidemic process on the complete graph $K_{N}$ with $N$ nodes. The infection and curing process are independent with infection rate $\beta$ and curing rate $\delta$, respectively. If we define $M(t)$ as the number of infected nodes at time $t$, then $M$ is a Markov process on $\{0,1, \ldots, N\}$ with the following rates:

$$
\begin{array}{llll}
M \mapsto M+1 & \text { at rate } & \beta M(N-M) \\
M \mapsto M-1 & \text { at rate } & \delta M .
\end{array}
$$

Indeed, every infected node heals with rate $\delta$, whereas every healthy node (of which there are $N-M$ ) has exactly $M$ infected neighbors.

We will analyze the critical $\tau=\frac{\beta}{\delta}$ as $N \rightarrow \infty$ by slightly altering the SIS Markov process: We propose that if the total number of infected sites reaches 1 , then that infected site cannot heal. In this way, the Markov process will not end in an absorbing state, and there will always exist a nondegenerate stationary state. Furthermore, as long as there are two or more infected nodes, the evolution of the two processes (SIS and modified SIS) is exactly the same. This means that if the MSIS has a stationary distribution for which we have very small probability of ending up in a state with $M=1$, this stationary distribution can be interpreted as a metastable state for the original SIS process. In the remainder of this article, we will consider the MSIS process, and with a slight abuse of notation, we will still use $M$ to denote the number of infected sites. Clearly, also for the MSIS process, $M$ is a Markov process on $\{1,2, \ldots, N\}$ with the following rates:

$$
\begin{array}{lll}
M \mapsto M+1 & \text { at rate } & \beta M(N-M) \\
M \mapsto M-1 & \text { at rate } & \delta M \times 1_{\{M \geqslant 2\}} .
\end{array}
$$

The stationary state of $M$ can be determined exactly. Let $\pi_{j}=\lim _{t \rightarrow \infty} \operatorname{Pr}[M(t)=j]$ be the steady-state probability that the number of infected nodes in the MSIS process equals $j$. In the steady state, the rate at which $M$ jumps from $i$ to $i+1$ must be equal to the rate at which $M$ jumps from $i+1$ to $i$, such that

$$
\forall 1 \leqslant i \leqslant N-1: \pi_{i} \beta i(N-i)=\pi_{i+1} \delta(i+1) .
$$

which is written, after iterating, in terms of the effective infection rate $\tau=\frac{\beta}{\delta}$ as

$$
\pi_{i+1}=\pi_{1} \frac{\tau^{i}}{i+1} \prod_{j=1}^{i}(N-j)=\pi_{1} \frac{\tau^{i}}{i+1} \frac{(N-1) !}{[N-(i+1)] !} .
$$

Using $\sum_{i=1}^{N} \pi_{i}=1$ and (1) leads to

$$
\frac{1}{\pi_{1}}=(N-1) ! \sum_{i=1}^{N} \frac{\tau^{i-1}}{i(N-i) !}=(N-1) ! \sum_{m=0}^{N-1} \frac{\tau^{N-m-1}}{(N-m) m !} .
$$

\section{A. The steady-state fraction of infected nodes}

The steady-state fraction of infected nodes, $y_{\infty ; N}(\tau)=$ $\frac{1}{N} \sum_{i=1}^{N} i \pi_{i}$, equals

$$
y_{\infty ; N}(\tau)=\frac{\frac{1}{N} \sum_{m=0}^{N-1} \frac{\tau^{-m}}{m !}}{\sum_{m=0}^{N-1} \frac{\tau^{-m}}{(N-m) m !}} .
$$


Clearly, $\lim _{\tau \rightarrow \infty} y_{\infty ; N}(\tau)=1$ and $^{1} \lim _{\tau \rightarrow 0} y_{\infty ; N}(\tau)=\frac{1}{N}$. Using $\frac{N}{N-m}=\frac{N-m+m}{N-m}$ in the denominator in Eq. (2) yields

$$
\begin{aligned}
\sum_{m=0}^{N-1} \frac{N \tau^{-m}}{(N-m) m !} & =\sum_{m=0}^{N-1} \frac{\tau^{-m}}{m !}+\sum_{m=0}^{N-1} \frac{m \tau^{-m}}{(N-m) m !} \\
& =\sum_{m=0}^{N-1} \frac{\tau^{-m}}{m !}+\frac{1}{\tau} \sum_{m=0}^{N-2} \frac{\tau^{-m}}{(N-1-m) m !}
\end{aligned}
$$

Now, using (2),

$$
\sum_{m=0}^{N-2} \frac{\tau^{-m}}{(N-1-m) m !}=\frac{\frac{1}{N-1} \sum_{m=0}^{N-2} \frac{\tau^{-m}}{m !}}{y_{\infty ; N-1}(\tau)}
$$

leads to the recursion

$$
y_{\infty ; N}(\tau)=\frac{1}{1+\frac{1}{(N-1) \tau y_{\infty ; N-1}(\tau)} \frac{\sum_{m=0}^{N-2} \frac{\tau^{-m}}{m !}}{\sum_{m=0}^{N-1} \frac{\tau^{-m}}{m !}}} .
$$

Rewritten with

$$
r_{N}(\tau)=\frac{\sum_{m=0}^{N-2} \frac{\tau^{-m}}{m !}}{\sum_{m=0}^{N-1} \frac{\tau^{-m}}{m !}}
$$

the recursion for the steady-state average number of infected nodes becomes

$$
y_{\infty ; N}(\tau)=1-\frac{r_{N}(\tau)}{(N-1) \tau} \frac{y_{\infty ; N}(\tau)}{y_{\infty ; N-1}(\tau)} .
$$

For sufficiently large $N$, there holds that $y_{\infty ; N}(\tau) \approx y_{\infty ; N-1}(\tau)$ and $r_{N}(\tau) \rightarrow 1$ and (3) simplifies, provided $(N-1) \tau>1$, to

$$
y_{\infty ; N}(\tau) \approx 1-\frac{1}{(N-1) \tau} .
$$

The steady-state fraction of infected nodes in NIMFA [9] for the complete graph $K_{N}$ equals, for $\tau \geqslant \tau_{c}^{(1)}=\frac{1}{N-1}$,

$$
y_{\infty ; N}^{(1)}(\tau)=1-\frac{1}{(N-1) \tau},
$$

illustrating how accurate NIMFA is for the complete graph.

\section{B. Asymptotics: Scaling law for large $N$}

We now determine the SIS epidemic behavior for large $N$ and deduce a scaling law for the epidemic threshold $\tau_{c}$ in the MSIS process. Since

$$
\begin{aligned}
\prod_{j=1}^{i}(N-j) & =N^{i} \prod_{j=1}^{i}\left(1-\frac{j}{N}\right)=N^{i} \exp \left[\sum_{j=1}^{i} \log \left(1-\frac{j}{N}\right)\right] \\
& =N^{i} \exp \left[-\sum_{j=1}^{i} \frac{j}{N}+O\left(i^{3} N^{-2}\right)\right] \\
& =N^{i} \exp \left[-\frac{i^{2}}{2 N}+O\left(i N^{-1}\right)+O\left(i^{3} N^{-2}\right)\right]
\end{aligned}
$$

\footnotetext{
${ }^{1}$ Multiply both numerator and denominator by $\tau^{N-1}$.
}

we rewrite (1) as

$$
\pi_{i+1}=\pi_{1} \frac{(\tau N)^{i}}{i+1} \exp \left(-\frac{1}{2} \frac{i^{2}}{N}\right)\left[1+O\left(i N^{-1}+i^{3} N^{-2}\right)\right] .
$$

In order to find what scaling for $\tau$ would still give metastable behavior (by choosing $\tau$ close to the critical value), we transform exponential factors in $i$ into a fully quadratic argument,

$$
\begin{aligned}
(\tau N)^{i} \exp \left(-\frac{1}{2} \frac{i^{2}}{N}\right) & =\exp \left(i \log \tau N-\frac{1}{2} \frac{i^{2}}{N}\right) \\
& =e^{\frac{N}{2}(\log \tau N)^{2}} \exp \left[-\frac{1}{2} \frac{(i-N \log \tau N)^{2}}{N}\right]
\end{aligned}
$$

and obtain

$$
\begin{aligned}
\pi_{i+1}= & \frac{\pi_{1} e^{\frac{N}{2}(\log \tau N)^{2}}}{i+1} \exp \left[-\frac{1}{2} \frac{(i-N \log \tau N)^{2}}{N}\right] \\
& \times\left[1+O\left(i N^{-1}+i^{3} N^{-2}\right)\right] .
\end{aligned}
$$

For large $N$, the appropriate scaling for $\tau$, for which we can still see metastable behavior, in the above expression of the probability $\pi_{i+1} \leqslant 1$ is found by requiring that $N(\log \tau N)^{2}=$ $c^{2}$, where $c$ is independent of $N$, which is equivalent to

$$
\tau=\frac{1}{N} \exp \left(\frac{c}{\sqrt{N}}\right)=\frac{1}{N}\left[1+\frac{c}{\sqrt{N}}+O\left(N^{-1}\right)\right]
$$

such that

$$
\begin{aligned}
\pi_{i+1}= & \frac{\pi_{1} e^{\frac{1}{2} c^{2}}}{i+1} \exp \left[-\frac{1}{2} \frac{(i-c \sqrt{N})^{2}}{N}\right] \\
& \times\left[1+O\left(i N^{-1}+i^{3} N^{-2}\right)\right] .
\end{aligned}
$$

Guided by the scaling for $\tau$, we define

$$
\tau=\frac{1+c / \sqrt{N}}{N}
$$

and proceed further with (4) to specify $c$. Then,

$$
\begin{aligned}
\pi_{i+1}= & \pi_{1} \frac{(1+c / \sqrt{N})^{i}}{i+1} \exp \left[-\frac{1}{2} \frac{(i+1)^{2}}{N}\right] \\
& \times\left[1+O\left(i N^{-1}+i^{3} N^{-2}\right)\right] .
\end{aligned}
$$

We can find $i_{\max }$ such that $\pi_{i+1}$ is maximal: If $c \geqslant 2$, then

$$
i_{\max }=\frac{c+\sqrt{c^{2}-4}}{2} \sqrt{N} .
$$

If $c<2$, then $i_{\max }=1$. Furthermore, the standard deviation of the stationary distribution is close to $\sqrt{N}$, more or less independent of $c$; this follows from inspecting (5), which shows that the stationary distribution of the MSIS process is close to a normal distribution with variance $N$. For a metastable state to exist, the MSIS distribution should have low probability of reaching only one infected site, which means that $\pi_{1}$ should be small. One way of ensuring a small $\pi_{1}$ could be to choose $c$ such that $i_{\max }$ is 3 times the standard deviation away from 1 . In other words, we choose $c$ such that

$$
\frac{1}{2}\left(c+\sqrt{c^{2}-4}\right)>3 \Leftrightarrow c>\frac{10}{3} .
$$


Of course, this choice is somewhat arbitrary, and other choices for a fixed critical $\tau$ could be made. We have to realize that the system shows metastable behavior, starting from $\tau$ values given by $\tau=\frac{1}{N}\left(1+\frac{c}{\sqrt{N}}\right)$ for large-enough $c$.

Finally, after summing (7) over $1 \leqslant i \leqslant N$ and using $\sum_{i=1}^{N} \pi_{i}=1$ and

$$
\sum_{i=1}^{N} \frac{(1+c / \sqrt{N})^{i-1}}{i} \exp \left(-\frac{1}{2} \frac{i^{2}}{N}\right)=\log (\sqrt{N})+O(1),
$$

we arrive at

$$
\pi_{i}=\frac{(1+c / \sqrt{N})^{i-1}}{i \log (\sqrt{N})} \exp \left(-\frac{1}{2} \frac{i^{2}}{N}\right)[1+o(1)],
$$

which illustrates that the probability of having one infected node in $K_{N}$ only decreases like $1 / \log (N)$ for any choice of $c$. Thus, (6) characterizes the correct scaling for epidemic threshold of the SIS metastable state in $K_{N}$. The expected number of infected nodes is

$$
\begin{aligned}
\sum_{i=1}^{N} i \pi_{i} & =\sum_{i=1}^{N} \frac{(1+c / \sqrt{N})^{i-1}}{\log (\sqrt{N})} \exp \left(-\frac{1}{2} \frac{i^{2}}{N}\right)[1+o(1)] \\
& =\frac{\sqrt{2 \pi N}}{\log (\sqrt{N})} \Phi(c) e^{\frac{1}{2} c^{2}}[1+o(1)] .
\end{aligned}
$$

where $\Phi$ is the standard normal distribution function.

The results of the MSIS model agree well with those of the $\varepsilon$-SIS model, in which each node can infect itself with rate $\varepsilon$ for a fixed $\varepsilon^{*}=\frac{\varepsilon}{\delta}<\frac{1}{N}$, as illustrated in Ref. [10].

\section{SIS EPIDEMICS ON THE STAR GRAPH}

The star graph consists of a center and $N$ "leaves."We define $X=1$ if the center is infected, and $X=0$ otherwise. Furthermore, we define $M$ as the number of leaves that are infected. Similarly to the complete graph, though more complicated, the number of infected nodes $M$ in the star can be reformulated as a SIS Markov chain with the following rates:

$$
\begin{aligned}
& (M, 0) \mapsto(M-1,0) \quad \text { at rate } M \delta, \\
& (M, 0) \mapsto(M, 1) \quad \text { at rate } M \beta, \\
& (M, 1) \mapsto(M-1,1) \quad \text { at rate } \quad M \delta, \\
& (M, 1) \mapsto(M+1,1) \quad \text { at rate } \quad(N-M) \beta, \\
& (M, 1) \mapsto(M, 0) \quad \text { at rate } \delta .
\end{aligned}
$$

Again we have an absorbing state if all nodes are healthy. We alter the chain slightly in order to obtain an irreducible Markov chain without an absorbing state.

The modified Markov chain without absorbing state, drawn in dotted box, is shown in Fig. 1. A state is characterized as $(M, X)$, where $M$ denotes the number of infected leaves of the star and $X=\{0,1\}$ indicates the infectious state of the hub or central node. Since we are concerned with the steady state, we can divide the Kolmogorov equations [11], deduced from the Markov graph, by $\delta$ to formulate the equation directly in terms of the effective infection rate $\tau=\frac{\beta}{\delta}$. In the sequel, we confine ourselves to the Markov chain, still denoted by $(M, X)$, but we make sure that when there is only one infected node. This

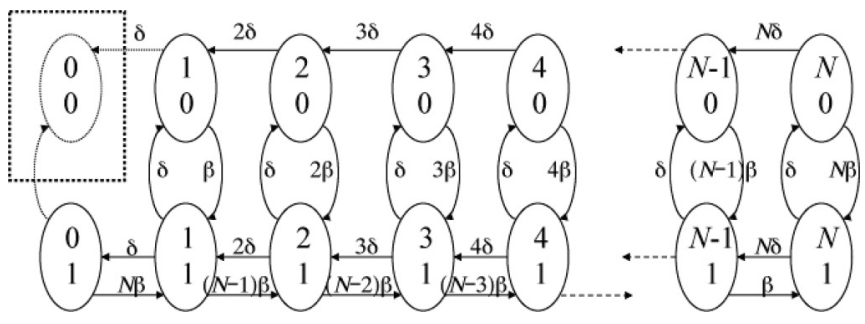

FIG. 1. The Markov graph for the SIS process on a star.

node cannot heal, so the rates become

$$
\begin{aligned}
& (M, 0) \mapsto(M-1,0) \quad \text { at rate } \quad M 1_{\{M \geqslant 2\}}, \\
& (M, 0) \mapsto(M, 1) \quad \text { at rate } \quad M \tau, \\
& (M, 1) \mapsto(M-1,1) \quad \text { at rate } \quad M, \\
& (M, 1) \mapsto(M+1,1) \quad \text { at rate } \quad(N-M) \tau, \\
& (M, 1) \mapsto(M, 0) \quad \text { at rate } \quad 1_{\{M \geqslant 1\}} .
\end{aligned}
$$

\section{A. Condition the star graph to have $X=1$}

To analyze the behavior of $(M, X)$, we start by ignoring the time spent by the chain on the set $X=0$ (when the center is healthy). This means that if we jump from state $(M, 1)$ to $(M, 0)$, we immediately move the chain to the point where it enters the set $X=1$ again. It turns out that we can determine this entry point, given that we exit at $M$ : when $X=0$, at each state $(i, 0)$, the process has probability $1 /(1+\tau)$ to jump to $(i-1,0)$ and probability $\tau /(1+\tau)$ to jump to $(i, 1)$. This means that the process will move a geometric number of steps to the left and then enter the set $X=1$ again. Of course, when the process walks all the way to $(1,0)$, thereafter it must go to $(1,1)$. Also, the process could enter $X=1$ at the same state as where it left $X=1$. We will ignore such transitions. We define this new Markov process on the set $\{0,1, \ldots, N\}$ as $M^{*}$ and we find the following jump rates for $M^{*}$, where we have to be careful at the edges of the set:

$$
\begin{aligned}
& M^{*} \mapsto M^{*}+1 \quad \text { at rate } \quad\left(N-M^{*}\right) \tau, \quad \text { at rate } 1_{\{j=1\}} M^{*} \\
& M^{*} \mapsto M^{*}-j \quad\left(\forall 1 \leqslant j \leqslant M^{*}-2\right), \\
&+\frac{\tau}{(1+\tau)^{j}} \quad(\forall 1 \leqslant)^{-M^{*}+1}, \\
& \forall M^{*} \geqslant 2: M^{*} \mapsto 1 \text { at rate } 1_{\left\{M^{*}=2\right\}} M^{*}+(1+\tau) \text { at rate } 1 .
\end{aligned}
$$

The general Markov theory now guarantees that if $\pi=$ $\left(\pi_{0}, \pi_{1}, \ldots, \pi_{N}\right)$ denotes the stationary measure or steady state for $M^{*}$, then $\pi$ equals the stationary state for $(M, X)$, conditioned to have $X=1$.

To determine the steady-state vector $\pi$, we look at the flow rates of the process from the set $\{i+1, \ldots, N\}$ to the set $\{0, \ldots, i\}$ and vice versa. Since these two rates should be equal in equilibrium, the following equations are found:

$$
\begin{aligned}
\forall 1 \leqslant i \leqslant N-1: \pi_{i}(N-i) \tau= & \pi_{i+1}\left(i+1+\frac{1}{1+\tau}\right) \\
& +\sum_{j=i+2}^{N} \frac{\pi_{j}}{(1+\tau)^{j-i}}
\end{aligned}
$$


and

$$
\pi_{1}=\pi_{0} \tau N
$$

The last equation is, of course,

$$
\sum_{i=0}^{N} \pi_{i}=1
$$

We transform these equations into a more elegant set. We rewrite equation (9) for $i-1$ and for $i$ but then multiplied by $1 /(1+\tau)$

$$
\begin{aligned}
\pi_{i-1}(N-i+1) \tau= & \pi_{i}\left(i+\frac{1}{1+\tau}\right)+\sum_{j=i+1}^{N} \frac{\pi_{j}}{(1+\tau)^{j-i+1}} \\
\frac{\pi_{i}(N-i) \tau}{1+\tau}= & \frac{\pi_{i+1}}{1+\tau}\left(i+1+\frac{1}{1+\tau}\right) \\
& +\sum_{j=i+2}^{N} \frac{\pi_{j}}{(1+\tau)^{j-i+1}} .
\end{aligned}
$$

Subtracting these two equations and multiplying by $1+\tau$ gives

$$
\begin{aligned}
& \pi_{i-1}(N-i+1) \tau(1+\tau)-\pi_{i}(N-i) \tau \\
& \quad=\pi_{i}(i+i \tau+1)-\pi_{i+1}\left(i+1+\frac{1}{1+\tau}\right)+\frac{\pi_{i+1}}{1+\tau},
\end{aligned}
$$

which leads to

$$
\begin{gathered}
\forall 2 \leqslant i \leqslant N-1:(i+1) \pi_{i+1}-(\tau N+i+1) \pi_{i} \\
+\tau(1+\tau)(N-i+1) \pi_{i-1}=0 .
\end{gathered}
$$

This set of equations can be solved exactly using a generating function approach as shown in Appendix A. Unfortunately, the resulting expressions (A16) for $\pi_{i}$ lack insight, which has led us to proceed with an asymptotic analysis (for large $N$ ).

\section{Asymptotic analysis}

The maximum of $\pi_{i}$ should be around $i=\tau N /(1+\tau)$. Indeed, put $\pi_{i-1}=\pi_{i}=\pi_{i+1}$ in Eq. (12), which corresponds to equating the discrete derivative to zero (we need three terms because we have a second-order equation). To analyze the solution of Eq. (12), we will assume that as $N \rightarrow \infty$, the $\pi_{i}$ 's can be rescaled to a smooth function in some way. We will scale around the location of the maximum by introducing a new variable $x \in \mathbb{R}$, which should replace $i$ :

$$
x(i)=N^{-\alpha}[(i-\tau N /(1+\tau)] .
$$

Here, $0<\alpha<1$ is a scaling parameter that we still have to choose. We then define the function $f$ by the following equation:

$$
\pi_{i}=N^{-\alpha} f\left[\frac{i-\tau N /(1+\tau)}{N^{\alpha}}\right]=N^{-\alpha} f(x(i)) .
$$

In this way,

$$
\begin{aligned}
\sum_{i=1}^{N} \pi_{i} & =\sum_{i=1}^{N} N^{-\alpha} f\left[\frac{i-\tau N /(1+\tau)}{N^{\alpha}}\right] \\
& \approx \int_{-\tau N^{1-\alpha}}^{\infty} f(x) d x,
\end{aligned}
$$

and if we pick the correct $\alpha$, we might end up with a nondegenerate density $f$ as $N \rightarrow \infty$. If such an $\alpha \in(0,1)$ exists, then for any other $\alpha^{\prime}$ we will obtain a degenerate result: If $\alpha^{\prime}>\alpha$, we zoom in too much and just find a constant equal to the maximal value of $f$, whereas if $\alpha^{\prime}<\alpha$, we zoom out too much, and we only see 0 . The correct $\alpha$ will depend on how we allow $\tau$ to change with $N$ : We are interested in finding a regime for $\tau$ where there still is a metastable phase. We therefore introduce two new constants, $C>0$ and $0 \leqslant \beta<1$, and choose $\tau=C N^{-\beta}$. Assuming that $f$ is smooth then leads to [denoting $x(i)$ with $x$ and simply using Taylor approximation]

$N^{\alpha} \pi_{i-1}=f(x)-N^{-\alpha} f^{\prime}(x)+\frac{1}{2} N^{-2 \alpha} f^{\prime \prime}(x)+$ lower order

and

$N^{\alpha} \pi_{i+1}=f(x)+N^{-\alpha} f^{\prime}(x)+\frac{1}{2} N^{-2 \alpha} f^{\prime \prime}(x)+$ lower order.

Equation (12) then becomes (after multiplying with $N^{\alpha}$ )

$$
\begin{aligned}
0= & -\tau(1+\tau) N^{\alpha} x f(x) \\
& +\left[-\frac{\tau^{2} N^{1-\alpha}}{1+\tau}+x+x \tau(1+\tau)\right] f^{\prime}(x) \\
& +\frac{1}{2}\left[\frac{N^{1-2 \alpha}\left(2 \tau+\tau^{2}\right)}{1+\tau}+x N^{-\alpha}-\tau(1+\tau) x N^{-\alpha}\right] f^{\prime \prime}(x) \\
& + \text { lower order. }
\end{aligned}
$$

(a) Regime $\beta<1 / 3$. It turns out that the system shows different behaviors for different choices of $\tau$. We start with supposing that $\beta<1 / 3$. If we take $\alpha=1 / 2-\beta / 2$, the highest order terms in Eq. (13) become

$$
\begin{aligned}
0 & =-\tau(1+\tau) N^{\alpha} x f(x)-\frac{\tau^{2} N^{1-\alpha}}{1+\tau} f^{\prime}(x) \\
& =\left[-C(1+\tau) x f(x)-\frac{C^{2}}{1+\tau} f^{\prime}(x)\right] N^{1 / 2-3 \beta / 2} .
\end{aligned}
$$

The above equation implies that $f$ is the density of a normal distribution with expectation 0 and variance $C /(1+\tau)^{2}$, so only for this choice of $\alpha$ will we get a nondegenerate result. This result is fairly easy to explain: If the center would be infected all the time (if we would forbid it to heal), then the leaves are all independent and at all times they would have probability $\tau /(1+\tau)$ to be infected. Since a sum of independent Bernoulli random variables tends to a Gaussian, the distribution of infected leaves tends to a normal (or Gaussian) distribution with expectation $\tau N /(1+\tau)$ and variance $(N \tau) /(1+\tau)^{2}=$ $N^{1-\beta} C /(1+\tau)^{2}$, which completely corresponds to our $f$ and our fluctuations of order $\alpha=1 / 2-\beta / 2$. Indeed, our scaling is set up such that if $M$ is the number of infected nodes, the variable $W=N^{-\alpha}[M-\tau N /(1+\tau)]$ has density $f$. Since $W$ is (approximately) normal with expectation 0 and variance $C /(1+\tau)^{2}$, we see that $M$ has a normal distribution with expectation $\tau N /(1+\tau)$ and variance $N^{2 \alpha} C /(1+\tau)^{2}$. If $\beta<1 / 3$, why would the distribution behave as if the center is always infected? The number of infected leaves is around $\tau N$, so the time for the healthy center to get infected again is of order $1 /\left(\tau^{2} N\right)$. In this time period, the number of healed leaves will be of order $\tau N /\left(\tau^{2} N\right)=1 / \tau$. Since $1 / \tau<N^{1 / 2-\beta / 2}$ (if $\beta<1 / 3$ ), the fluctuations caused by the center being healthy are negligible compared to the fluctuations when the center is 
always infected. Therefore, when $\beta<1 / 3$, the system behaves as if the center is always infected.

(b) Regime $\beta>1 / 3$. The case $\beta>1 / 3$ behaves differently. If we again choose $\alpha=1 / 2-\beta / 2$, we would now have that the highest-order terms in Eq. (13) become

$$
0=x f^{\prime}(x)+C f^{\prime \prime}(x) .
$$

The solution which satisfies $\lim _{x \rightarrow \infty} f(x)=0$ is given by

$$
f(x)=A[1-\Phi(x / \sqrt{C})],
$$

with $\Phi$ the distribution function of the standard normal distribution. The behavior for $x \rightarrow-\infty$ is a bit puzzling: The function $f$ does not go to 0 . The reason is that to the left of $\tau N$, $\pi_{i}$ is decreasing at a rate slower than $\alpha=1 / 2-\beta / 2$. In other words, when $\beta<1 / 3$, the stable distribution is symmetric (even normal) around its maximum, but when $\beta>1 / 3$, the distribution becomes very skewed as $N \rightarrow \infty$, where the left tail is much heavier than the right tail. For the regime $x<0$, it turns out that in order to get a sensible differential equation for $f$, we need to choose $\alpha=\beta$. The highest-order terms in Eq. (13) when $x<0$ are then given by

$$
0=-C x f(x)+x f^{\prime}(x) .
$$

When $x$ is very close to 0, other terms in Eq. (13) start playing a role. The solution is given by

$$
f(x)=B e^{C x} .
$$

The constant $B$ is determined by the fact that $f$ is a density: It should hold that

$$
\int_{-\tau N^{1-\alpha}}^{0} B e^{C x} d x=1 .
$$

We ignore the $N^{1 / 2-\beta / 2}$ regime, since it is much smaller than the $N^{\beta}$ regime: This means that $f$ is going to 0 when $x \rightarrow \infty$ at such a fast rate that the positive half line does not contribute significantly to $\int_{\mathbb{R}} f(x) d x$. If $\beta<1 / 2$, we get $B=C+o(1)$, and if $\beta=1 / 2$, then

$$
B=\frac{C}{1-e^{-C^{2}}} .
$$

We have found that if $1 / 3<\beta<1 / 2$ and $\tau=C N^{-\beta}$, then for $x<0$ and $i=\tau N+x N^{\beta}$ we obtain

$$
\pi_{i}=C e^{C x} N^{-\beta}[1+o(1)] .
$$

For $\beta=1 / 2, \tau=C / \sqrt{N}, x<0$ and $i=\tau N+x \sqrt{N}$, we get

$$
\pi_{i}=\frac{C e^{C x}}{1-e^{-C^{2}}} \frac{1}{\sqrt{N}}[1+o(1)] .
$$

For $x>0$, we find that $\sqrt{N} \pi_{i} \rightarrow 0$. It seems that $\beta=1 / 2$ corresponds to the critical $\tau$, since, in that case, the fluctuations are of the same order as $\tau N$. This means that there is a reasonable chance of approaching the absorbing state in which all nodes are healthy. However, it will turn out that this chance will actually go to 1 .

\section{B. Full stationary distribution for the star graph}

In the previous section, we have determined asymptotically the stationary distribution of the SIS epidemics on the star graph, conditioned to be on the set $\{X=1\}$, that is, conditioned to have an infected center. Now define $\rho_{i}$ as the stationary measure conditioned on the set $\{X=0\}$, where the index $i \in[1, N]$ as illustrated in Fig. 1 . Furthermore, let $p$ be the probability that the stationary distribution has an infected center. Together, $\pi_{i}, \rho_{i}$, and $p$ determine the full stationary measure or steady state.

From Fig. 1, we can write down the detailed balance equation for the state $(M=i, X=0)$ with $2 \leqslant i \leqslant N-1$,

$$
(1-p) \rho_{i}(1+\tau) i=(1-p) \rho_{i+1}(i+1)+p \pi_{i} .
$$

We can solve $\rho_{i}$ exactly for $2 \leqslant i \leqslant N$,

$$
i \rho_{i}=\sum_{j=i}^{N} \frac{p}{1-p} \pi_{j} \frac{1}{(1+\tau)^{j+1-i}} .
$$

This solution satisfies the boundary condition

$$
(1-p) \rho_{N}(1+\tau) N=p \pi_{N},
$$

which exactly corresponds to the detailed balance equation for the state $(M=N, X=0)$. We also know that

$$
(1-p) \rho_{1} \tau=2(1-p) \rho_{2}+p \pi_{1},
$$

from which

$$
\rho_{1}=\frac{2}{\tau} \rho_{2}+\frac{p}{1-p} \pi_{1}
$$

The latter equation is very relevant, because $\rho_{1}$ is a factor $\tau^{-1}$ larger than $\rho_{2}$ and might dominate all other values $\rho_{i}$ (so it might happen that $\rho_{1} \rightarrow 1$ ). Finally, the normalization of the steady-state probabilities, conditioned that the center is healthy $(X=0)$, is

$$
\sum_{i=1}^{N} \rho_{i}=1
$$

In addition, we need to determine $p$, the probability that the center is infected. If we look at the rate of going from the set $\{X=0\}$ to the set $\{X=1\}$, and vice versa, we find

$$
\sum_{i=1}^{N} p \pi_{i}=\sum_{i=1}^{N}(1-p) \rho_{i} i \tau .
$$

Solving for $p$, using (11) and the definition

$$
\mathbb{E} \rho=\sum_{i=1}^{N} \rho_{i} i
$$

yields

$$
p=\frac{\tau \mathbb{E} \rho}{1-\pi_{0}+\tau \mathbb{E} \rho},
$$

where $\pi_{0}$ is the steady-state probability of being in state ( $M=0, X=1)$, determined by the detailed balance equation (10). In fact, $\pi_{0}$ will always tend to zero as follows from Eq. (10): Whenever $\tau N \rightarrow \infty$, and when $\tau N=O(1)$, the time the system resides in the state where only the center is infected is $N$ times as short as the time the system resides in the state where only one leave is infected. 


\section{Asymptotic analysis of the two $\beta$ regimes}

(a) Regime $\beta<1 / 3$. If $\beta<1 / 3$ and $\tau=C N^{-\beta}$, we have already seen that, when the center becomes healthy, the number of leaves that heal during the healthy period is negligible. Therefore, $\rho_{i}=\pi_{i}[1+o(1)]$. Furthermore, the center heals at rate 1 , whereas it becomes infected with rate $\tau N /(1+\tau)[1+$ $o(1)]$ (i.e., proportional to the number of infected leaves), so

$$
p=\frac{\tau N}{1+\tau+\tau N}[1+o(1)]=\left(1-\frac{1+\tau}{\tau N}\right)[1+o(1)]
$$

and $p \rightarrow 1$ when $\beta<1 / 3$. This means that the center is almost always infected, as we have seen before.

(b) Regime $\beta>1 / 3$. Now we investigate the case where $1 / 3<\beta \leqslant 1 / 2$. We recall (14) and (15) to conclude that there exists a constant $B^{*}$ such that

$$
\pi_{i}=B^{*} e^{\tau i}[1+o(1)]
$$

for $i \leqslant \tau N$ and that $\pi_{i}$ is of smaller order for larger $i$. If $i>$ $\tau N$, then $\pi_{i}=0$ in the scaling $\alpha=\beta>\frac{1}{3}$. Using these values of $\pi_{i}$ in Eq. (16) and evaluating the resulting geometric series, it follows that there exists a $D>0$ such that, for $2 \leqslant i \leqslant \tau N$,

$$
i \rho_{i}=D[1+o(1)]\left[\frac{e^{\tau^{2} N}}{(1+\tau)^{\tau N}}(1+\tau)^{i}-e^{i \tau}\right] .
$$

Define $\rho_{i}^{*}$, for $2 \leqslant i \leqslant \tau N$, by

$$
i \rho_{i}^{*}=\frac{e^{\tau^{2} N}}{(1+\tau)^{\tau N}}(1+\tau)^{i}-e^{i \tau}
$$

So

$$
\rho_{i}=\left\{\begin{array}{cl}
D \rho_{i}^{*} & \text { for } 2 \leqslant i \leqslant \tau N \\
0 & \text { for } i>\tau N
\end{array} .\right.
$$

We need an estimate of $\mathbb{E} \rho$. Since $\rho_{i}=D \rho_{i}^{*}$, we first look at

$$
\begin{aligned}
\sum_{i=2}^{\tau N} i \rho_{i}^{*} & =\frac{e^{\tau^{2} N}}{(1+\tau)^{\tau N}} \sum_{i=2}^{\tau N}(1+\tau)^{i}-\sum_{i=2}^{\tau N} e^{i \tau}=\frac{e^{\tau^{2} N}}{(1+\tau)^{\tau N}} \frac{(1+\tau)^{\tau N+1}-(1+\tau)^{2}}{\tau}-\frac{e^{\tau^{2} N+\tau}-e^{2 \tau}}{e^{\tau}-1} \\
& =e^{\tau^{2} N}\left(\frac{1+\tau}{\tau}-\frac{e^{\tau}}{e^{\tau}-1}\right)-\left[\frac{(1+\tau)^{-\tau N+2} e^{\tau^{2} N}}{\tau}-\frac{e^{2 \tau}}{e^{\tau}-1}\right] \\
& =\frac{1}{2}[1+o(1)] e^{\tau^{2} N}-\frac{\exp \left[\tau^{2} N-(\tau N-2) \log (1+\tau)\right]-e^{2 \tau}}{\tau}-\frac{1}{2}[1+o(1)]
\end{aligned}
$$

and

$$
\sum_{i=2}^{\tau N} i \rho_{i}^{*}=\frac{1}{2}\left[e^{\tau^{2} N}-1-\tau^{2} N\right][1+o(1)]
$$

Further, we have that

$$
\rho_{2}^{*}=\frac{1}{2}\left[\frac{e^{\tau^{2} N}}{(1+\tau)^{\tau N}}(1+\tau)^{2}-e^{2 \tau}\right]=\frac{1}{2}\left[e^{\tau^{2} N-(\tau N-2) \log (1+\tau)}-e^{2 \tau}\right]=\frac{1}{2}\left[e^{\frac{1}{2} \tau^{3} N+o\left(\tau^{3} N\right)}-1\right][1+o(1)]
$$

and

$$
\rho_{2}^{*}=\frac{1}{4} \tau^{3} N[1+o(1)] .
$$

Note from $\tau=C N^{-\beta}$ that $\tau^{3} N \rightarrow 0$ since $\beta>1 / 3$. Ignoring $1+o(1)$ factors, recalling that $\rho_{i}^{*}=0$ for $i>\tau N$ and combining equations (17) and (19)-(21), we have

$$
\mathbb{E} \rho=\sum_{i=1}^{N} \rho_{i} i=D \sum_{i=1}^{N \tau} \rho_{i}^{*} i=\frac{1}{2} D\left(e^{\tau^{2} N}-1-\tau^{2} N\right)+\frac{2}{\tau} D \frac{1}{4} \tau^{3} N+\frac{p}{1-p} \pi_{1}=\frac{1}{2} D\left(e^{\tau^{2} N}-1\right)+\tau \mathbb{E} \rho \pi_{1},
$$

which implies that

$$
\mathbb{E} \rho=\frac{1}{2} D\left(e^{\tau^{2} N}-1\right)[1+o(1)]
$$

It remains to determine $D$, which can be achieved from $\sum_{i=1}^{N} \rho_{i}=1$. We first determine

$$
\begin{aligned}
\sum_{i=2}^{\tau N} \rho_{i}^{*} & =[1+o(1)] \int_{2}^{\tau N}\left[e^{\tau^{2} N+(x-\tau N) \log (1+\tau)}-e^{x \tau}\right] \frac{d x}{x}=[1+o(1)] \int_{2}^{\tau N} e^{x \tau}\left(e^{-\frac{1}{2} x \tau^{2}+\frac{1}{2} \tau^{3} N}-1\right) \frac{d x}{x} \\
& =[1+o(1)] \frac{1}{2} \tau^{3} N \int_{2 / \tau N}^{1} e^{s \tau^{2} N} \frac{1-s}{s} d s=[1+o(1)] \frac{1}{2} \tau^{3} N\left(\int_{2 / \tau N}^{1} \frac{d s}{s}+\int_{0}^{1} \frac{e^{s \tau^{2} N}-s e^{s \tau^{2} N}-1}{s} d s\right) \\
& =[1+o(1)] \frac{1}{2} \tau^{3} N\left(\log (\tau N)+\int_{0}^{1} \frac{e^{s \tau^{2} N}-s e^{s \tau^{2} N}-1}{s} d s\right)
\end{aligned}
$$


and, after some tedious calculations, we arrive at

$$
\sum_{i=2}^{\tau N} \rho_{i}^{*}=\frac{1}{2}\left[\tau^{3} N \log (\tau N)+\frac{e^{\tau^{2} N}}{\tau N}\right][1+o(1)] .
$$

Since $\sum_{i=\tau N+1}^{N} \rho_{i}^{*}=0$ because $\rho_{i}^{*}=0$ for $i>\tau N$, combining (17), (22), and (21) leads to

$$
\begin{aligned}
1 & =\rho_{1}+\sum_{i=2}^{N} \rho_{i}=\frac{2 \rho_{2}}{\tau}+\frac{p}{1-p} \pi_{1}+\frac{1}{2} D\left[\tau^{3} N \log (\tau N)+\frac{e^{\tau^{2} N}}{\tau N}\right][1+o(1)] \\
& =\left\{\frac{1}{2} \tau^{2} N+\frac{1}{2}\left[\tau^{3} N \log (\tau N)+\frac{e^{\tau^{2} N}}{\tau N}\right]\right\} D[1+o(1)]+\tau \mathbb{E} \rho \pi_{1} \\
& =\left\{\frac{1}{2} \tau^{2} N+\frac{1}{2}\left[\tau^{3} N \log (\tau N)+\frac{e^{\tau^{2} N}}{\tau N}\right]+\frac{1}{2} \tau\left(e^{\tau^{2} N}-1\right) \pi_{1}\right\} D[1+o(1)],
\end{aligned}
$$

from which

$$
D=\frac{1+o(1)}{\frac{1}{2} \tau^{2} N+\frac{1}{2} \tau\left(e^{\tau^{2} N}-1\right) \pi_{1}+\frac{1}{2} \tau^{3} N \log (\tau N)+\frac{1}{2} e^{\tau^{2} N}(\tau N)^{-1}}
$$

\section{Asymptotic scaling of $\tau$}

Let us now investigate the scaling for $\tau$. If $\tau=C / \sqrt{N}$ in Eq. (23),

$$
D=\frac{1+o(1)}{\frac{1}{2} C^{2}+\frac{1}{2} \frac{C}{\sqrt{N}}\left(e^{C^{2}}-1\right) \pi_{1}+\frac{1}{2} \frac{C^{3}}{\sqrt{N}} \log (C \sqrt{N})+\frac{1}{2} \frac{e^{C^{2}}}{C \sqrt{N}},}
$$

then, for large $N \rightarrow \infty$, we find

$$
D=\frac{1+o(1)}{\frac{1}{2} C^{2}}
$$

and

$$
\mathbb{E} \rho=\frac{e^{C^{2}}-1}{C^{2}}[1+o(1)]
$$

so

$$
p=\frac{C / \sqrt{N} \mathbb{E} \rho}{1-\pi_{0}+C / \sqrt{N} \mathbb{E} \rho},
$$

which implies that $p \rightarrow 0$ (the center is almost always healthy) and $\rho_{1} \rightarrow 1$. Clearly this means that in the original SIS model, the system will quickly heal as $N$ grows larger.

To ensure that we stay away from the healthy state, a minimal requirement would be that $\rho_{1} \rightarrow 0$. Relation (17) reveals that the first term dominates the second term whenever $\tau>1 / N$, and therefore, also using (21),

$$
\rho_{1}=\frac{1}{2} \tau^{2} N D[1+o(1)] .
$$

Thus, we need a slightly larger $\tau$ than $\frac{C}{\sqrt{N}}$, so

$$
\tau^{2} N D \rightarrow 0
$$

Since the fourth term in the denominator of Eq. (23) will be the dominant term, this can be accomplished precisely by choosing $\tau$ such that

$$
\frac{e^{\tau^{2} N}}{\tau^{3} N^{2}} \rightarrow \infty
$$

A bit of puzzling with logarithmic corrections then leads to the choice

$$
\tau=\frac{1}{\sqrt{N}} \sqrt{\frac{1}{2} \log (N)+\frac{3}{2} \log (\log (N))+a_{n}},
$$

where $a_{n}$ is any sequence going to $+\infty$, no matter how slowly. Using this $\tau$ in Eq. (24) indeed gives

$$
\begin{aligned}
& \frac{e^{\frac{1}{2} \log (N)+\frac{3}{2} \log (\log (N))+a_{n}}}{N^{1 / 2}\left[\frac{1}{2} \log (N)+\frac{3}{2} \log (\log (N))+a_{n}\right]^{3 / 2}} \\
& =\frac{\log (N)^{\frac{3}{2}} e^{a_{n}}}{\left[\frac{1}{2} \log (N)+\frac{3}{2} \log (\log (N))+a_{n}\right]^{3 / 2}} \\
& \rightarrow \infty .
\end{aligned}
$$

With the choice for $\tau$ in Eq. (25), we find that $p \rightarrow 1$ and $\rho_{1} \rightarrow 0$, but very slowly. In summary, it is reasonable to define the epidemic threshold in the star as

$$
\tau_{c}=\frac{1}{\sqrt{N}} \sqrt{\frac{1}{2} \log (N)+\frac{3}{2} \log (\log (N))} .
$$

Any $\tau$ significantly larger than $\tau_{c}$ (meaning that $\tau / \tau_{c} \rightarrow \infty$ ) would lead to a metastable state. Moreover, $(25)$ indicates that the difference between a critical $\tau_{c}$ and $C / \sqrt{N}$ is very hard to distinguish, for some $C>0$ and finite $N$.

\section{Comparison with the mean-field approximation}

The steady-state fraction of infected nodes in the star (with $N+1$ nodes) in NIMFA [9] equals

$$
y_{\infty}^{(1)}(\tau)=\frac{\left(N-\tau^{-2}\right)}{N+1}\left\{\frac{1}{\tau^{-1}+1}+\frac{1}{\tau^{-1}+N}\right\} .
$$

The exact computation of the steady-state fraction $y_{\infty}(\tau)$ of infected nodes in the star for any $N$ is based on the Markov 


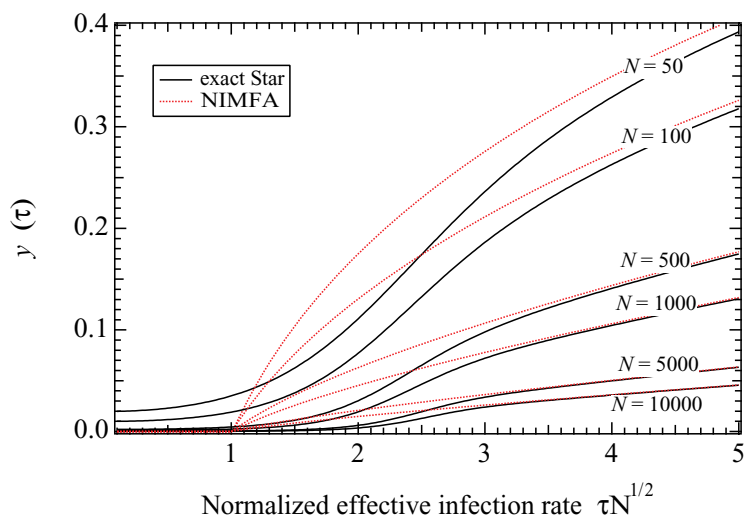

FIG. 2. (Color online) The steady-state fraction $y_{\infty}(\tau)$ versus the normalized effected infection rate $\tau \sqrt{N}$ for various sizes of $N$ of the exact and the $N$-intertwined mean-field approximation. All meanfield curves have an onset of infection precisely starting at $\tau \sqrt{N}=1$, clearly lower bounding the exact onset of the epidemic in the star.

equations (12) with (10), (11) for $\pi_{i}$ and (16) and (17) for $\rho_{i}$, and (19) for $p$. Figure 2 illustrates the difference between the exact steady-state fraction $y_{\infty}(\tau)$ of infected nodes in the star and that in NIMFA (27).

For larger $N$ and larger $\tau$, both curves in Fig. 2 coincide. However, for larger $N$, the onset of the SIS epidemic shifts to increasingly larger values of the normalized effective infection rate $\tau \sqrt{N}$, indicating that $\tau_{c}$ does not scale as $\frac{C}{\sqrt{N}}$ but slightly faster as $\tau_{c}=\alpha \tau_{c}^{(1)}$, where $\alpha=\sqrt{\frac{1}{2} \log N+\frac{3}{2} \log \log N}$ and $\tau_{c}^{(1)}=\frac{1}{\sqrt{N}}$ according to Eq. (26).

\section{CONCLUSION}

Since the metastable (or quasistationary regime) in SIS epidemics is not precisely defined for finite sizes of the graph, two different approaches are proposed in this paper: (a) a generalization of the SIS model to the $\varepsilon$-SIS model in Ref. [10] and (b) a modified SIS model that prevents the epidemics to die out. For the complete graph $K_{N}$, both approaches are analytically shown to lead to precisely the same epidemic threshold scaling with $N$. Moreover, NIMFA is extremely good for the complete graph; in fact, among all graphs, NIMFA is most accurate for $K_{N}$. For the star graph, on the other hand, the analysis, culminating in Fig. 2 and the precise scaling law (25) for the epidemic threshold $\tau_{c}$, shows larger discrepancies between the exact MSIS model and NIMFA. In particular, the onset of the epidemic is smeared out (for finite $N$ ) without a sharp transition point. In addition, the convergence with $N$ towards such a sharp threshold $\tau_{c}$ is rather slow.
The star graph represents a limiting case of a scale-free or power-law degree distribution. In addition, the hub structure, that many complex networks possess, can be regarded as a set of sparsely interconnected large stars. From this perspective, the epidemics in the star exhibits a bounding or limiting behavior of the SIS epidemics in power law graphs. The SIS epidemic in the power law graphs with different degree exponents was studied in detail by Pastor-Satoras and Castellano in several papers, such as Refs. [12] and [13] (and other references therein), mainly simulative, although the role of the central hub (via the star graph) was analyzed approximately in Ref. [12]. Our exact asymptotic analysis of the star, which reflects two distinct asymptotic $\tau$ regimes, may shed light on the local initial onset of the SIS epidemics in scale-free graphs (with degree power exponent $\gamma>3$ ), followed by a propagation of the epidemics to the rest of the network.

\section{APPENDIX: SOLUTION OF THE DIFFERENCE EQUATION (12)}

We consider the set of difference equations (12) that determine the set $\left\{\pi_{k}\right\}_{0 \leqslant k \leqslant N}$,

$$
\begin{aligned}
\forall 2 \leqslant i \leqslant N-1: & (i+1) \pi_{i+1}-(i+\tau N+1) \pi_{i} \\
& +\tau(1+\tau)(N-i+1) \pi_{i-1}=0 .
\end{aligned}
$$

with (10) and (11) and the $i=N-1$ equation from Eq. (9),

$$
\pi_{N-1}=\frac{\pi_{N}}{\tau}\left(N+\frac{1}{1+\tau}\right) .
$$

\section{Differential equation for the generating function}

We define the generating function $G(z)=\sum_{k=0}^{N} \pi_{k} z^{k}$ with $\pi_{k}=0$ when $k \notin[0, N]$, which satisfies $G(1)=1$, due to Eq. (11). Multiplying (12) by $z^{i}$ and summing over all $2 \leqslant i \leqslant N-1$ yields

$$
\begin{aligned}
& \sum_{i=2}^{N-1}(i+1) \pi_{i+1} z^{i}-\sum_{i=2}^{N-1}(i+\tau N+1) \pi_{i} z^{i} \\
& \quad+\tau(1+\tau) \sum_{i=2}^{N-1}(N-i+1) \pi_{i-1} z^{i}=0 .
\end{aligned}
$$

We consider each summation separately. First, using $G^{\prime}(z)=$ $\sum_{k=1}^{N} k \pi_{k} z^{k-1}$, we have

$$
\begin{aligned}
\sum_{i=2}^{N-1}(i+1) \pi_{i+1} z^{i} & =\sum_{i=3}^{N} i \pi_{i} z^{i-1}=\sum_{i=1}^{N} i \pi_{i} z^{i-1}-2 \pi_{2} z-\pi_{1} \\
& =G^{\prime}(z)-2 \pi_{2} z-\pi_{1} .
\end{aligned}
$$

The second summation is

$$
\begin{aligned}
\sum_{i=2}^{N-1}(i+\tau N+1) \pi_{i} z^{i} & =\sum_{i=1}^{N} i \pi_{i} z^{i}-\pi_{1} z-N \pi_{N} z^{N}+(\tau N+1)\left(\sum_{i=0}^{N} \pi_{i} z^{i}-\pi_{0}-\pi_{1} z-\pi_{N} z^{N}\right) \\
& =z G^{\prime}(z)+(\tau N+1) G(z)-(\tau N+1) \pi_{0}-(\tau N+2) \pi_{1} z-[N(\tau+1)+1] \pi_{N} z^{N},
\end{aligned}
$$


while the third summation equals

$$
\begin{aligned}
& \sum_{i=2}^{N-1}(N-i+1) \pi_{i-1} z^{i} \\
& \quad=\sum_{i=1}^{N-2}(N-i) \pi_{i} z^{i+1} \\
& \quad=\sum_{i=1}^{N}(N-i) \pi_{i} z^{i+1}-\pi_{N-1} z^{N} \\
& =N z G(z)-z^{2} G^{\prime}(z)-N z \pi_{0}-\pi_{N-1} z^{N} .
\end{aligned}
$$

Combining all pieces results in the governing equation for the generating function

$$
\begin{aligned}
& {\left[1-z-\tau(1+\tau) z^{2}\right] G^{\prime}(z)+[\tau(1+\tau) N z-(\tau N+1)] G(z)} \\
& \quad=R(z)
\end{aligned}
$$

where

$$
\begin{aligned}
R(z)= & \pi_{1}-(\tau N+1) \pi_{0} \\
& +\left\{2 \pi_{2}-(\tau N+2) \pi_{1}+\tau(1+\tau) N \pi_{0}\right\} z \\
& \left.-\{[N(\tau+1)+1)] \pi_{N}-\tau(1+\tau) \pi_{N-1}\right\} z^{N} .
\end{aligned}
$$

Using $\pi_{1}=\pi_{0} \tau N$ from Eq. (10) and $\pi_{N-1}=\frac{\pi_{N}}{\tau}\left(N+\frac{1}{1+\tau}\right)$ from Eq. (A1) yields

$$
R(z)=-\pi_{0}+\left\{2 \pi_{2}-\tau N[\tau N+1-\tau] \pi_{0}\right\} z,
$$

which is linear in $z$. Observe the ominous appearence of $\pi_{2}$, because there is only the additional condition $G(1)=1$, while two unkowns $\pi_{0}$ and $\pi_{2}$ need to be determined.

It follows from Eq. (A2) that, for $z=1$ and using $G(1)=1$,

$$
[-\tau(1+\tau)] G^{\prime}(1)+\tau(1+\tau) N-(\tau N+1)=R(1) .
$$

Hence, the average number of infected nodes in the conditioned Markov chain in the steady state, $G^{\prime}(1)=\sum_{k=1}^{N} k \pi_{k}$, equals

$$
G^{\prime}(1)=\frac{\left(\tau^{2} N-1\right)+[1+\tau N(\tau N+1-\tau)] \pi_{0}-2 \pi_{2}}{\tau(1+\tau)} .
$$

\section{a. Homogeneous solution of (A2)}

We first concentrate on the homogenous solution of the differential equation (A2), from which the logarithmic derivative is

$$
\frac{G_{h}^{\prime}(z)}{G_{h}(z)}=\frac{\tau(1+\tau) N z-(\tau N+1)}{\tau(1+\tau) z^{2}+z-1} .
$$

The integration of the right-hand side requires the partial fraction decomposition,

$$
\frac{\tau(1+\tau) N z-(\tau N+1)}{\tau(1+\tau) z^{2}+z-1}=\frac{c_{1}}{z-r_{1}}+\frac{c_{2}}{z-r_{2}},
$$

where $r_{1}$ and $r_{2}$ are the zeros of the quadratic polynomial $\tau(1+\tau) z^{2}+z-1$ and $c_{1,2}$ are the corresponding residues.
Thus,

$$
\tau(1+\tau) z^{2}+z-1=\tau(1+\tau)\left(z-r_{1}\right)\left(z-r_{2}\right),
$$

where

$$
\begin{aligned}
& r_{1}=\frac{-1+\sqrt{1+4 \tau(1+\tau)}}{2 \tau(1+\tau)}=\frac{1}{1+\tau}>0 \\
& r_{2}=\frac{-1-\sqrt{1+4 \tau(1+\tau)}}{2 \tau(1+\tau)}=-\frac{1}{\tau}<0
\end{aligned}
$$

with $r_{1} r_{2}=r_{1}+r_{2}=-\frac{1}{\tau(1+\tau)}$. The residues $c_{k}$ for $k=1,2$ follow from

$$
c_{k}=\lim _{z \rightarrow r_{k}} \frac{\left(N z-\frac{\tau N+1}{\tau(1+\tau)}\right)\left(z-r_{k}\right)}{\left(z-r_{1}\right)\left(z-r_{2}\right)} .
$$

In addition, since $\frac{c_{1}}{z-r_{1}}+\frac{c_{2}}{z-r_{2}}=\frac{\left(c_{1}+c_{2}\right) z-\left(c_{1} r_{2}+c_{2} r_{1}\right)}{\left(z-r_{1}\right)\left(z-r_{2}\right)}=\frac{N z-\frac{\tau N+1}{\tau(1+\tau)}}{z^{2}+\frac{z-1}{\tau(1+\tau)}}$, we have that

$$
\begin{aligned}
c_{1}+c_{2} & =N \\
c_{1} r_{2}+c_{2} r_{1} & =\frac{\tau N+1}{\tau(1+\tau)},
\end{aligned}
$$

from which $c_{1}=N-c_{2}$ and

$$
c_{1}=\frac{N r_{1}-\frac{\tau N+1}{\tau(1+\tau)}}{r_{1}-r_{2}}=-\frac{1}{1+2 \tau} .
$$

Hence, for $\tau \geqslant 0$, we find that $-1 \leqslant c_{1} \leqslant 0$ and $c_{1}$ is only an integer for either $\tau=0$ or $\tau \rightarrow \infty$. Since $c_{2}=N-c_{1}$, we have that $N \leqslant c_{2} \leqslant N+1$.

Integration of Eq. (A5) yields

$$
\log G_{h}(z)=c_{1} \log \left(z-r_{1}\right)+c_{2} \log \left(z-r_{2}\right)+b,
$$

where $b$ is an integration constant. Finally, the homogeneous solution of Eq. (A2) is

$$
G_{h}(z)=e^{b}\left(z-r_{1}\right)^{c_{1}}\left(z-r_{2}\right)^{N-c_{1}} .
$$

Explicitly,

$$
G_{h}(z)=e^{b} \frac{\left(z+\frac{1}{\tau}\right)^{N+\frac{1}{1+2 \tau}}}{\left(z-\frac{1}{1+\tau}\right)^{\frac{1}{1+2 \tau}}}=e^{b}\left(z+\frac{1}{\tau}\right)^{N}\left(\frac{z+\frac{1}{\tau}}{z-\frac{1}{1+\tau}}\right)^{\frac{1}{1+2 \tau}}
$$

illustrates that $G_{h}(z)$ has an essential singularity for $z=\frac{1}{1+\tau}$ and a branch cut for $\operatorname{Re} z<\frac{1}{1+\tau}$, because $\left(z-\frac{1}{1+\tau}\right)^{\frac{1}{1+2 \tau}}=$ $e^{\frac{\log \left(z-\frac{1}{1+\tau}\right)}{1+2 \tau}}$. Also the numerator has a branch cut for $\operatorname{Re} z<-\frac{1}{\tau}$. In other words, $G_{h}(z)$ is not analytic around $z=0$ and not in the disk $|z|<1$, whereas analyticity for $|z|<1$ is required for any probability generating function.

\section{b. Particular solution of (A2)}

The particular solution of the differential equation (A2) is found by the method of the variation of a constant, where

$$
G(z)=B(z)\left(z-r_{1}\right)^{c_{1}}\left(z-r_{2}\right)^{N-c_{1}}
$$


and

$$
\begin{aligned}
G^{\prime}(z)= & B^{\prime}(z)\left(z-r_{1}\right)^{c_{1}}\left(z-r_{2}\right)^{N-c_{1}} \\
& +c_{1} B(z)\left(z-r_{1}\right)^{c_{1}-1}\left(z-r_{2}\right)^{N-c_{1}} \\
& +\left(N-c_{1}\right) B(z)\left(z-r_{1}\right)^{c_{1}}\left(z-r_{2}\right)^{N-c_{1}-1} .
\end{aligned}
$$

Introduced into (A2) yields

$$
\begin{aligned}
R(z)= & -\tau(1+\tau) B^{\prime}(z)\left(z-r_{1}\right)^{c_{1}+1}\left(z-r_{2}\right)^{N-c_{1}+1} \\
& +(\tau(1+\tau) N z-(\tau N+1)-\tau(1+\tau)) \\
& \times\left\{N z-c_{1} r_{2}-\left(N-c_{1}\right) r_{1}\right\} \\
& \times B(z)\left(z-r_{1}\right)^{c_{1}}\left(z-r_{2}\right)^{N-c_{1}} .
\end{aligned}
$$

The coefficient of the last term vanishes (due to the relation between the residues),

$$
R(z)=-\tau(1+\tau) B^{\prime}(z)\left(z-r_{1}\right)^{c_{1}+1}\left(z-r_{2}\right)^{N-c_{1}+1}
$$

or

$$
B^{\prime}(z)=\frac{R(z)}{-\tau(1+\tau)\left(z-r_{1}\right)^{c_{1}+1}\left(z-r_{2}\right)^{N-c_{1}+1}},
$$

where $R(z)=b z+c \quad$ with $\quad c=-\pi_{0} \quad$ and $\quad b=2 \pi_{2}-$ $\tau N[\tau N+1-\tau] \pi_{0}$. After integrating (A9), we have

$$
B(z)=B\left(z_{0}\right)-\frac{1}{\tau(1+\tau)} \int_{z_{0}}^{z} \frac{R(u)}{\left(u-r_{1}\right)^{c_{1}+1}\left(u-r_{2}\right)^{N-c_{1}+1}} d u .
$$

An appropriate choice for $z_{0}=1$, because $B(1)$ is known from Eq. (A6), by invoking the condition $G(1)=1$, as

$$
B(1)=\left(\frac{\tau}{1+\tau}\right)^{N-2 c_{1}}
$$

Combining (A6) and (A10), the general solution of Eq. (A2) is

$$
\begin{aligned}
G(z)= & B(1)\left(z-r_{1}\right)^{c_{1}}\left(z-r_{2}\right)^{N-c_{1}} \\
& -\frac{1}{\tau(1+\tau)} \int_{1}^{z} \frac{\left(z-r_{1}\right)^{c_{1}}\left(z-r_{2}\right)^{N-c_{1}} R(u)}{\left(u-r_{1}\right)^{c_{1}+1}\left(u-r_{2}\right)^{N-c_{1}+1}} d u,
\end{aligned}
$$

where the first function possesses a branch cut for $\operatorname{Re}(z)<$ $r_{1}=\frac{1}{1+\tau}$. Using the expressions for $r_{1}, r_{2}$, and $c_{1}$, the integral in Eq. (A12), denoted by $I(z)$, becomes

$$
\begin{aligned}
I(z)= & \frac{1}{\tau(1+\tau)} \int_{1}^{z}\left(\frac{u-\frac{1}{1+\tau}}{u+\frac{1}{\tau}}\right)^{\frac{1}{1+2 \tau}} \\
& \times \frac{b u+c}{\left(u-\frac{1}{1+\tau}\right)\left(u+\frac{1}{\tau}\right)^{N+1}} d u,
\end{aligned}
$$

which can, as shown in Sec. A 2, be written in terms of incomplete Beta functions.

\section{The integral $I(z)$}

Since

$$
\frac{u-\frac{1}{1+\tau}}{u+\frac{1}{\tau}}=\frac{u+\frac{1}{\tau}-\frac{1}{1+\tau}-\frac{1}{\tau}}{u+\frac{1}{\tau}}=1-\frac{1+2 \tau}{\tau(1+\tau)\left(u+\frac{1}{\tau}\right)},
$$

the integral $I(z)$ in Eq. (A13) is

$$
\begin{aligned}
I(z)= & \frac{1}{\tau(1+\tau)} \int_{1}^{z}\left[1-\frac{1+2 \tau}{\tau(1+\tau)\left(u+\frac{1}{\tau}\right)}\right]^{\frac{1}{1+2 \tau}} \\
& \times \frac{b u+c}{\left(u-\frac{1}{1+\tau}\right)\left(u+\frac{1}{\tau}\right)^{N+1}} d u .
\end{aligned}
$$

We now make a series of substitutions in the integration variable. First, let $x=u+\frac{1}{\tau}$, then

$$
\begin{aligned}
I(z)= & \frac{1}{\tau(1+\tau)} \int_{1+\frac{1}{\tau}}^{z+\frac{1}{\tau}}\left(1-\frac{1+2 \tau}{\tau(1+\tau) x}\right)^{\frac{1}{1+2 \tau}-1} \\
& \times \frac{b x+c-\frac{b}{\tau}}{x^{N+2}} d x .
\end{aligned}
$$

Next, we put $y=\frac{1}{x}$ and obtain

$$
\begin{aligned}
I(z)= & -\frac{1}{\tau(1+\tau)} \int_{\frac{\tau}{1+\tau}}^{\frac{\tau}{1+z \tau}}\left[1-\frac{1+2 \tau}{\tau(1+\tau)} y\right]^{\frac{1}{1+2 \tau}-1} \\
& \times\left(b / y+c-\frac{b}{\tau}\right) y^{N} d y .
\end{aligned}
$$

Finally, let $w=\frac{1+2 \tau}{\tau(1+\tau)} y$,

$$
I(z)=-\frac{1}{1+2 \tau}\left[\frac{\tau(1+\tau)}{1+2 \tau}\right]^{N} \int_{\frac{1+2 \tau}{(1+\tau)^{2}}}^{\frac{1+2 \tau}{(1+\tau)(1+z \tau)}}(1-w)^{\frac{1}{1+2 \tau}-1}\left[\frac{1+2 \tau}{\tau(1+\tau)} \frac{b}{w}+c-\frac{b}{\tau}\right] w^{N} d w
$$

and

$$
I(z)=-\frac{b}{\tau(1+\tau)}\left[\frac{\tau(1+\tau)}{1+2 \tau}\right]^{N} \int_{\frac{1+2 \tau}{(1+\tau)^{2}}}^{\frac{1+2 \tau}{(1+\tau)(1+z \tau)}}(1-w)^{\frac{1}{1+2 \tau}-1} w^{N-1} d w-\frac{\left(c-\frac{b}{\tau}\right)}{1+2 \tau}\left[\frac{\tau(1+\tau)}{1+2 \tau}\right]_{\frac{1+2 \tau}{(1+\tau)^{2}}}^{N} \int^{\frac{1+2 \tau}{(1+\tau)(1+z \tau)}}(1-w)^{\frac{1}{1+2 \tau}-1} w^{N} d w .
$$

The remaining integrals can be written in terms of the incomplete Beta function $B(x ; a, b)=\int_{0}^{x} t^{a-1}(1-t)^{b-1} d t$ as

$$
\int_{\alpha}^{x} w^{m}(1-w)^{q-1} d w=\int_{0}^{x} w^{m}(1-w)^{q-1} d w-\int_{0}^{\alpha} w^{m}(1-w)^{q-1} d w=B(x, m+1, q)-B(\alpha, m+1, q),
$$


where $\alpha<1$. If $x=\frac{1+2 \tau}{(1+\tau)(1+z \tau)}$ exceeds 1 (thus $z<\frac{1}{1+\tau}$ ), the integrand, in particular $(1-t)^{q-1}$, possesses a branch cut. In summary,

$$
\begin{aligned}
I(z)= & -\frac{b}{\tau(1+\tau)}\left[\frac{\tau(1+\tau)}{1+2 \tau}\right]^{N}\left\{B\left[\frac{1+2 \tau}{(1+\tau)(1+z \tau)}, N, \frac{1}{1+2 \tau}\right]-B\left[\frac{1+2 \tau}{(1+\tau)^{2}}, N, \frac{1}{1+2 \tau}\right]\right\} \\
& -\frac{\left(c-\frac{b}{\tau}\right)}{1+2 \tau}\left[\frac{\tau(1+\tau)}{1+2 \tau}\right]^{N}\left\{B\left[\frac{1+2 \tau}{(1+\tau)(1+z \tau)}, N+1, \frac{1}{1+2 \tau}\right]-B\left[\frac{1+2 \tau}{(1+\tau)^{2}}, N+1, \frac{1}{1+2 \tau}\right]\right\} .
\end{aligned}
$$

Using the exact evaluation for $a \in \mathbb{N}$,

$$
B(x ; a, b)=\frac{\Gamma(a) \Gamma(b)}{\Gamma(b+a)}-\Gamma(a) \Gamma(b) \sum_{j=0}^{a-1} \frac{x^{a-1-j}(1-x)^{j+b}}{\Gamma(a-j) \Gamma(b+j+1)},
$$

derived by repeated partial integration, and

$$
\left(z-r_{1}\right)^{c_{1}}\left(z-r_{2}\right)^{N-c_{1}}=\tau^{-N}\left[1-\frac{1+2 \tau}{(1+\tau)(\tau z+1)}\right]^{-\frac{1}{1+2 \tau}}(1+z \tau)^{N}
$$

we have that

$$
\begin{aligned}
s_{1} & =\left(z-r_{1}\right)^{c_{1}}\left(z-r_{2}\right)^{N-c_{1}}\left\{B\left[\frac{1+2 \tau}{(1+\tau)(1+z \tau)}, N, \frac{1}{1+2 \tau}\right]-B\left[\frac{1+2 \tau}{(1+\tau)^{2}}, N, \frac{1}{1+2 \tau}\right]\right\} \\
& =-(N-1) ! \tau^{-N}(1+z \tau)\left(\frac{1+2 \tau}{1+\tau}\right)^{N-1} \sum_{j=0}^{N-1} \frac{\Gamma\left(\frac{1}{1+2 \tau}\right)\left[\frac{(1+\tau)(1+z \tau)}{1+2 \tau}-1\right]^{j}}{\Gamma\left(\frac{1}{1+2 \tau}+j+1\right)(N-1-j) !}+\left(z-r_{1}\right)^{c_{1}}\left(z-r_{2}\right)^{N-c_{1}} q_{1},
\end{aligned}
$$

where $q_{1}=q(N-1)$ and

$$
q(N)=N ! \Gamma\left(\frac{1}{1+2 \tau}\right)\left[\frac{1+2 \tau}{(1+\tau)^{2}}\right]^{N}\left[\frac{\tau^{2}}{(1+\tau)^{2}}\right]^{\frac{1}{1+2 \tau}} \sum_{j=0}^{N} \frac{\left(\frac{\tau^{2}}{1+2 \tau}\right)^{j}}{(N-j) ! \Gamma\left(\frac{1}{1+2 \tau}+j+1\right)},
$$

illustrating that the first sum is analytic in $z$ everywhere, while the second sum is not analytic inside $|z|<1$. Similarly,

$$
\begin{aligned}
s_{2} & =\left(z-r_{1}\right)^{c_{1}}\left(z-r_{2}\right)^{N-c_{1}}\left\{B\left[\frac{1+2 \tau}{(1+\tau)(1+z \tau)}, N+1, \frac{1}{1+2 \tau}\right]-B\left[\frac{1+2 \tau}{(1+\tau)^{2}}, N+1, \frac{1}{1+2 \tau}\right]\right\} \\
& =-N ! \tau^{-N-1}\left(\frac{1+2 \tau}{1+\tau}\right)^{N} \sum_{j=0}^{N} \frac{\Gamma\left(\frac{1}{1+2 \tau}\right)\left[\frac{(1+\tau)(1+z \tau)}{1+2 \tau}-1\right]^{j}}{\Gamma\left(\frac{1}{1+2 \tau}+j+1\right)(N-j) !}+\left(z-r_{1}\right)^{c_{1}}\left(z-r_{2}\right)^{N-c_{1}} q_{2},
\end{aligned}
$$

where $q_{2}=q(N)$.

\section{Determination of $G(z)$ and $\pi_{m}$}

With these new definitions, the expression in Eq. (A12) for $G(z)$ becomes

$$
\begin{aligned}
G(z) & =B(1)\left(z-r_{1}\right)^{c_{1}}\left(z-r_{2}\right)^{N-c_{1}}-\left(z-r_{1}\right)^{c_{1}}\left(z-r_{2}\right)^{N-c_{1}} I(z) \\
& =B(1)\left(z-r_{1}\right)^{c_{1}}\left(z-r_{2}\right)^{N-c_{1}}+\frac{b}{\tau(1+\tau)}\left[\frac{\tau(1+\tau)}{1+2 \tau}\right]^{N} s_{1}+\frac{\left(c-\frac{b}{\tau}\right)}{1+2 \tau}\left[\frac{\tau(1+\tau)}{1+2 \tau}\right]^{N} s_{2} .
\end{aligned}
$$

In order for $G(z)=\sum_{k=0}^{N} \pi_{k} z^{k}$ to be a probability-generating function (and, in our case, analytic everwhere), we must require that

$$
B(1)+\frac{b}{\tau(1+\tau)}\left[\frac{\tau(1+\tau)}{1+2 \tau}\right]^{N} q_{1}+\frac{\left(c-\frac{b}{\tau}\right)}{1+2 \tau}\left[\frac{\tau(1+\tau)}{1+2 \tau}\right]^{N} q_{2}=0 .
$$

With (A11), recall that $b=2 \pi_{2}-\tau N[\tau N+1-\tau] \pi_{0}$ and $c=-\pi_{0}$, we find that

$$
b=\frac{\tau}{\frac{q_{1}}{(1+\tau)}-\frac{q_{2}}{(1+2 \tau)}}\left\{\frac{-q_{2}}{1+2 \tau} c-\left[\frac{1+2 \tau}{(1+\tau)^{2}}\right]^{N}\left(\frac{\tau}{1+\tau}\right)^{\frac{2}{1+2 \tau}}\right\} .
$$


With this requirement, we arrive at

$$
G(z)=-\frac{b}{\tau} \frac{(N-1) !}{1+2 \tau}(1+z \tau) \sum_{j=0}^{N-1} \frac{\Gamma\left(\frac{1}{1+2 \tau}\right)\left[\frac{(1+\tau)(1+z \tau)}{1+2 \tau}-1\right]^{j}}{\Gamma\left(\frac{1}{1+2 \tau}+j+1\right)(N-1-j) !}-\frac{\left(c-\frac{b}{\tau}\right) N !}{1+2 \tau} \frac{1}{\tau} \sum_{j=0}^{N} \frac{\Gamma\left(\frac{1}{1+2 \tau}\right)\left[\frac{(1+\tau)(1+z \tau)}{1+2 \tau}-1\right]^{j}}{\Gamma\left(\frac{1}{1+2 \tau}+j+1\right)(N-j) !} .
$$

We expand $\left[\frac{(1+\tau)(1+z \tau)}{1+2 \tau}-1\right]^{j}=(-1)^{j}\left(\frac{\tau}{1+2 \tau}\right)^{j}[1-z(1+\tau)]^{j}$ into a Taylor series around $z=0$,

$$
\left[\frac{(1+\tau)(1+z \tau)}{1+2 \tau}-1\right]^{j}=\left(\frac{\tau}{1+2 \tau}\right)^{j} \sum_{m=0}^{j}\left(\begin{array}{c}
j \\
m
\end{array}\right)(-1)^{j-m}(1+\tau)^{m} z^{m}
$$

Substituted into the above relation for $G(z)$ yields after summation reversal,

$$
\begin{aligned}
G(z)= & -\frac{b}{\tau} \frac{(N-1) !}{1+2 \tau} \sum_{m=0}^{N-1}\left\{\sum_{j=m}^{N-1} \frac{(-1)^{j-m}\left(\begin{array}{c}
j \\
m
\end{array}\right) \Gamma\left(\frac{1}{1+2 \tau}\right)\left(\frac{\tau}{1+2 \tau}\right)^{j}}{\Gamma\left(\frac{1}{1+2 \tau}+j+1\right)(N-1-j) !}\right\}(1+\tau)^{m} z^{m} \\
& -b \frac{(N-1) !}{1+2 \tau} \sum_{m=1}^{N}\left\{\sum_{j=m-1}^{N-1} \frac{(-1)^{j-m-1}\left(\begin{array}{c}
j \\
m-1
\end{array}\right) \Gamma\left(\frac{1}{1+2 \tau}\right)\left(\frac{\tau}{1+2 \tau}\right)^{j}}{\Gamma\left(\frac{1}{1+2 \tau}+j+1\right)(N-1-j) !}\right\}(1+\tau)^{m-1} z^{m} \\
& -\frac{\left(c-\frac{b}{\tau}\right) N !}{1+2 \tau} \frac{1}{\tau} \sum_{m=0}^{N}\left\{\sum_{j=m}^{N} \frac{(-1)^{j-m}\left(\begin{array}{c}
j \\
m
\end{array}\right) \Gamma\left(\frac{1}{1+2 \tau}\right)\left(\frac{\tau}{1+2 \tau}\right)^{j}}{\Gamma\left(\frac{1}{1+2 \tau}+j+1\right)(N-j) !}\right\}(1+\tau)^{m} z^{m} .
\end{aligned}
$$

After equating corresponding powers of $z$ in $G(z)=\sum_{k=0}^{N} \pi_{k} z^{k}$ and the above expression, this leads to

$$
\pi_{0}=-\frac{b}{\tau} \frac{(N-1) !}{1+2 \tau}\left\{\sum_{j=0}^{N-1} \frac{(-1)^{j} \Gamma\left(\frac{1}{1+2 \tau}\right)\left(\frac{\tau}{1+2 \tau}\right)^{j}}{\Gamma\left(\frac{1}{1+2 \tau}+j+1\right)(N-1-j) !}\right\}-\frac{\left(c-\frac{b}{\tau}\right) N !}{1+2 \tau} \frac{1}{\tau}\left\{\sum_{j=0}^{N} \frac{(-1)^{j} \Gamma\left(\frac{1}{1+2 \tau}\right)\left(\frac{\tau}{1+2 \tau}\right)^{j}}{\Gamma\left(\frac{1}{1+2 \tau}+j+1\right)(N-j) !}\right\}
$$

and, for $1 \leqslant m \leqslant N$,

$$
\begin{aligned}
\pi_{m}= & -\frac{b}{\tau} \frac{(N-1) !}{1+2 \tau}(1+\tau)^{m}\left\{\sum_{j=m}^{N-1} \frac{(-1)^{j-m}\left(\begin{array}{c}
j \\
m
\end{array}\right) \Gamma\left(\frac{1}{1+2 \tau}\right)\left(\frac{\tau}{1+2 \tau}\right)^{j}}{\Gamma\left(\frac{1}{1+2 \tau}+j+1\right)(N-1-j) !}\right\} \\
& -b \frac{(N-1) !}{1+2 \tau}(1+\tau)^{m-1}\left\{\sum_{j=m-1}^{N-1} \frac{(-1)^{j-m-1}\left(\begin{array}{c}
j \\
m-1
\end{array}\right) \Gamma\left(\frac{1}{1+2 \tau}\right)\left(\frac{\tau}{1+2 \tau}\right)^{j}}{\Gamma\left(\frac{1}{1+2 \tau}+j+1\right)(N-1-j) !}\right\} \\
& -\frac{\left(c-\frac{b}{\tau}\right) N !}{1+2 \tau} \frac{1}{\tau}(1+\tau)^{m}\left\{\sum_{j=m}^{N} \frac{(-1)^{j-m}\left(\begin{array}{c}
j \\
m
\end{array}\right) \Gamma\left(\frac{1}{1+2 \tau}\right)\left(\frac{\tau}{1+2 \tau}\right)^{j}}{\Gamma\left(\frac{1}{1+2 \tau}+j+1\right)(N-j) !}\right\} .
\end{aligned}
$$

It remains to determine $b$ and $c$ from the above expression for $\pi_{0}$ and from the analyticity condition (A15). Substituting (A15) into the expression of $\pi_{0}$ and recalling that $c=-\pi_{0}$ yields

$$
\begin{aligned}
\pi_{0}= & \frac{(N-1) ! v_{1}}{q_{2}-\frac{\left(1+2 \tau q_{1}\right.}{(1+\tau)}}\left\{\frac{q_{2}}{1+2 \tau} \pi_{0}-\left[\frac{1+2 \tau}{(1+\tau)^{2}}\right]^{N}\left(\frac{\tau}{1+\tau}\right)^{\frac{2}{1+2 \tau}}\right\}+\frac{\pi_{0} N !}{1+2 \tau} \frac{v_{2}}{\tau} \\
& -\frac{N ! \frac{1}{\tau} v_{2}}{q_{2}-\frac{(1+2 \tau) q_{1}}{(1+\tau)}}\left\{\frac{q_{2}}{1+2 \tau} \pi_{0}-\left[\frac{1+2 \tau}{(1+\tau)^{2}}\right]^{N}\left(\frac{\tau}{1+\tau}\right)^{\frac{2}{1+2 \tau}}\right\}
\end{aligned}
$$

with $v_{1}=v(N-1)$ and $v_{2}=v(N)$, where

$$
v(N)=\sum_{j=0}^{N} \frac{(-1)^{j} \Gamma\left(\frac{1}{1+2 \tau}\right)\left(\frac{\tau}{1+2 \tau}\right)^{j}}{\Gamma\left(\frac{1}{1+2 \tau}+j+1\right)(N-j) !} .
$$

Solving for $\pi_{0}$ finally results in

$$
\pi_{0}=\frac{\left[\frac{1+2 \tau}{(1+\tau)^{2}}\right]^{N}\left(\frac{\tau}{1+\tau}\right)^{\frac{2}{1+2 \tau}}(N-1) !\left(\frac{N}{\tau} v_{2}-v_{1}\right)}{\left(1-\frac{N !}{1+2 \tau} \frac{v_{2}}{\tau}\right)\left[q_{2}-\frac{(1+2 \tau) q_{1}}{1+\tau}\right]+\frac{(N-1) ! q_{2}\left(\frac{N}{\tau} v_{2}-v_{1}\right)}{1+2 \tau}} .
$$


From Eq. (A17), $c=-\pi_{0}$ and $b$ in Eq. (A15) follow and, therefore, all probabilities $\pi_{m}$ for $0 \leqslant m \leqslant N$. In addition, $G^{\prime}(1)$ is elegantly computed from Eq. (A4) as

$$
G^{\prime}(1)=\frac{\left(\tau^{2} N-1\right)-(b+c)}{\tau(1+\tau)},
$$

avoiding the determination of all $\pi_{m}$.

[1] R. M. Anderson and R. M. May, Infectious Diseases of Humans: Dynamics and Control (Oxford University Press, Oxford, UK, 1991).

[2] D. J. Daley and J. Gani, Epidemic Modeling: An Introduction (Cambridge University Press, Cambridge, UK, 1999).

[3] P. Van Mieghem, J. Omic, and R. E. Kooij, IEEE/ACM Transact. Network. 17, 1 (2009).

[4] R. Durrett, Proc. Natl. Acad. Sci. USA 107, 4491 (2010).

[5] P. L. Simon, M. Taylor, and I. Z. Kiss, Math. Biol. 62, 479 (2011).

[6] R. Pastor-Satorras and A. Vespignani, Phys. Rev. E 63, 066117 (2001).
[7] C. Li, R. van de Bovenkamp, and P. Van Mieghem, Phys. Rev. E 86, 026116 (2012).

[8] E. Cator and P. Van Mieghem, Phys. Rev. E 85, 056111 (2012).

[9] P. Van Mieghem, Computing 93, 147 (2011).

[10] P. Van Mieghem and E. Cator, Phys. Rev. E 86, 016116 (2012).

[11] P. Van Mieghem, Performance Analysis of Communications Systems and Networks (Cambridge University Press, Cambridge, UK, 2006).

[12] C. Castellano and R. Pastor-Satorras, Competing activation mechanisms in epidemics on networks. Nature Scientific Reports, 2(371), April 2012.

[13] C. Castellano and R. Pastor-Satorras, Phys. Rev. Lett. 105, 218701 (2010). 